\title{
Hsa_circ_0016760 exacerbates the malignant development of non-small cell lung cancer by sponging miR-145-5p/FGF5
}

\author{
ZHENG ZHU, QIYONG WU, MING ZHANG, JICHUN TONG, BIN ZHONG and KAI YUAN \\ Department of Cardiothoracic Surgery, The Affiliated Changzhou No. 2 People's Hospital of \\ Nanjing Medical University, Changzhou, Jiangsu 213000, P.R. China
}

Received June 16, 2020; Accepted October 13, 2020

DOI: $10.3892 /$ or.2020.7899

\begin{abstract}
Hsa_circ_0016760 expression has been reported to be increased in non-small cell lung cancer (NSCLC). The present study was designed to explore the role and mechanism of hsa_circ_0016760 in regulating NSCLC progression. In total, 60NSCLC patients were followed-up for 60 months after surgery. Hsa_circ_0016760 expression in tumor tissues and adjacent non-tumor tissues of NSCLC patients was explored by reverse transcription quantitative polymerase chain reaction (RT-qPCR). NSCLC cell proliferation was monitored by CCK-8 assay and EdU experiment. Transwell assays were used for the detection of NSCLC cell migration and invasion. The target of hsa_circ_0016760 (or miR-145-5p) was validated by luciferase reporter gene assay and RNA immunoprecipitation experiment. A xenograft model was studied with nude mice. Immunohistochemical staining was applied for the detection of $\mathrm{Ki} 67$ expression in xenograft tumors. Hsa_circ_0016760/miR-145-5p/FGF5 expression in tissues and cells was investigated by RT-qPCR and western blotting. Hsa_circ_0016760 was aberrantly upregulated in NSCLC, which was associated with poor prognosis of patients $(\mathrm{P}<0.05)$. Hsa_circ_0016760 silencing suppressed NSCLC cell proliferation, migration and invasion in vitro $(\mathrm{P}<0.01)$. Hsa_circ_0016760 facilitated FGF5 expression via sponging miR-145-5p. The miR-145-5p upregulation or FGF5 downregulation reversed the promoting effect of hsa_circ_0016760 on NSCLC cell proliferation, migration and invasion in vitro $(\mathrm{P}<0.01)$. In addition, hsa_circ_0016760 silencing inhibited tumor growth in vivo $(\mathrm{P}<0.01)$, and decreased Ki67 expression in xenograft tumors. In conclusion, hsa_circ_0016760 exacerbated the malignant development of NSCLC by sponging miR-145-5p/FGF5.
\end{abstract}

Correspondence to: Dr Kai Yuan, Department of Cardiothoracic Surgery, The Affiliated Changzhou No. 2 People's Hospital of Nanjing Medical University, 29 Xinglong Lane, Changzhou, Jiangsu 213000, P.R. China

E-mail: dr_yuankai@163.com

Key words: non-small cell lung cancer, hsa_circ_0016760, miR-145-5p/FGF5, progression, in vivo study

\section{Introduction}

Lung cancer remains one of the leading causes of cancer-related deaths worldwide. As the most common and aggressive type of lung cancer, the incidence of non-small cell lung cancer (NSCLC) accounts for approximately 85\% (1). Clinically, surgical resection and postoperative adjuvant therapy, including chemotherapy and radiotherapy, remain the primary choice of treatment for NSCLC patients at early stages. However, a considerable proportion of NSCLC patients are in advanced stages or the cancer has metastasized at the time of diagnosis. Thereby, a great number of patients with advanced NSCLC cannot benefit from surgical resection (2). Hence, the 5-year survival rate of NSCLC patients remains markedly poor despite the great progress that has been made in the past few years in chemotherapy and radiotherapy treatments (3). Therefore, the elucidation of the mechanism involved in the progression of NSCLC is essential to improve the prognosis of patients.

Circular RNAs (circRNAs) are commonly expressed in eukaryotic cells, and are associated with the progression of human tumors $(4,5)$. However, limited research has focused on the underlying mechanism of circRNAs in regulating the development of NSCLC. Through circRNA microarrays, a previous research screened hsa_circ_0016760 as a tumor-specific circRNA candidate towards NSCLC tissues and adjacent normal tissues (6). Furthermore, Li et al (7) revealed the enhanced expression of hsa_circ_0016760 in NSCLC tissues and cells, and that high expression of hsa_circ_0016760 was closely related to the poor prognosis of patients as well as lymph node metastasis and advanced TNM stages. The upregulated hsa_circ_0016760 could facilitate NSCLC cell growth and metastatic properties in vitro. In terms of mechanism, the carcinogenic properties of circ_0016760 were attributed to the enhancement of GAGE1 expression via sponging miR-1287. To date, more studies concerning the function of circ_0016760 in NSCLC or other human tumors are scarce.

Hence, the present study was designed to explore the effect of circ_0016760 in regulating NSCLC progression in order to provide an effective theoretical basis for the application of circ_0016760 in the targeted therapy of NSCLC. In terms of the molecular mechanism network, it was observed from biological software online analysis that miR-145-5p possessed binding sites for circ_0016760 and FGF5. Previous studies 
have reported that miR-145-5p and FGF5 are involved in the progression of lung cancer $(8,9)$. Thus, the present study researched the mechanism of circ_0016760 in regulating NSCLC with the miR-145-5p/FGF5 axis.

\section{Materials and methods}

Patients and tissues. NSCLC patients $(\mathrm{n}=80)$ who were treated at our hospital (the Affiliated Changzhou No. 2 People's Hospital of Nanjing Medical University, Changzhou, Jiangsu) from August 2013 to October 2014 were enrolled in the present study. All patients were diagnosed with NSCLC for the first time and had no previous history of cancer-related treatment. The inclusion criteria were as follows: i) Patients who voluntarily joined the study; ii) patients who were diagnosed with NSCLC for the first time; iii) patients without previous treatment history related to cancer diseases.

The exclusion criteria were as follows: i) Patients who did not want to join this study; ii) patients with previous treatment history of cancer-related diseases. Surgical resection was performed on these patients. During surgery, tumor tissues and adjacent normal tissues were collected and stored at $-80^{\circ} \mathrm{C}$. The clinical characteristics of all patients are recorded and listed in Table I. All patients were followed-up for 60 months after surgery.

All patients volunteered to join the study and written informed consent was signed by all subjects. The present study complied with the Declaration of Helsinki and was approved by the Ethics Committee of the Affiliated Changzhou No. 2 People's Hospital of Nanjing Medical University.

Cell culture. Six NSCLC cell lines (NCI-H1395, A549, H460, H1975, H1299 and Calu3) and normal human bronchial epithelial cell line (NHBE) were obtained from the Institute of Biochemistry and Cell Biology of the Chinese Academy of Sciences. Cells were grown in Dulbecco's modified Eagle's medium (DMEM) supplemented with $10 \%$ (v/v) fetal bovine serum (FBS) in a humidified incubator at $37^{\circ} \mathrm{C}$ and $5 \% \mathrm{CO}_{2}$.

Structural stability test of hsa_circ_0016760. Tumor tissues collected from NSCLC patients were ground into powder in liquid nitrogen. Total RNA in tumor tissues were extracted with TRIzol reagent (Thermo Fisher Scientific, Inc.). A total of $5.0 \mu \mathrm{g}$ of total RNA sample was subjected to incubation with 10 units RNase R for $30 \mathrm{~min}$ at $37^{\circ} \mathrm{C}$ (used as the RNase $\mathrm{R}$ group). In addition, $5.0 \mu \mathrm{g}$ of total RNA sample without any treatment was collected and used as the Mock group. The expression of hsa_circ_0016760 and glyceraldehyde-3-phosphate dehydrogenase (GAPDH) in the two groups was monitored through reverse transcription quantitative polymerase chain reaction (RT-qPCR). It is well known that the structure of linear RNA is easily destroyed by RNase R, whereas the circular structure of circular RNA is difficult to be degraded by RNase $\mathrm{R}$ (10). Therefore, the circular structure stability of hsa_circ_0016760 could be detected.

Cell transfection. In this experiment, Lipofectamine 2000 reagent (Thermo Fisher Scientific, Inc.) was used for transfection. $\mathrm{H} 1299$ and Calu3 cells $\left(1 \times 10^{6}\right.$ cells $\left./ \mathrm{ml} ; 1 \mathrm{ml}\right)$ cultured in 6-well plates with serum-free DMEM were transfected by small interfering RNA (100 nM; siRNA-1: 5'-GUCUGGCAUGCAGAGGCAGAA-3', siRNA-2: 5'-CUG GCAUGCAGAGGCAGAAGA-3' and siRNA-3: 5'-AUG CAGAGGCAGAAGAGGCCU-3', respectively) targeting hsa_circ_0016760 (named the si-hsa_circ_0016760-1 group, si-hsa_circ_0016760-2 group and si-hsa_circ_0016760-3 group, respectively). The transfection was performed at $37^{\circ} \mathrm{C}$ for $8 \mathrm{~h}$. $\mathrm{H} 1299$ and Calu3 cells transfected by siRNA negative control (100 nM; 5'-AGGAGGCUAGCUCUGCGACUU-3') were used as the si-Ctrl groups. The miR-145-5p mimic (100 nM; miR-145-5p mimic group, sequence: 5'-GUCCAG UUUUCCCAGGAAUCCCU-3') and mimic negative control (100 nM; miR-NC group, sequence: 5'-CGCGAGUUAACG GACCAUACGGU-3') were used to transfect H1299 and Calu3 cells. siRNA and corresponding negative control, miR-145-5p mimic and mimic negative control were synthesized by Shanghai GenePharma Co., Ltd.

pCDNA3.1 vector containing the full length of hsa_circ_0016760 was provided by Shanghai Genechem Co., Ltd., and was then transfected into A549 cells $\left(1 \times 10^{6}\right.$ cells $\left./ \mathrm{ml} ; 1 \mathrm{ml}\right)$ cultured in 6-well plates with serum-free DMEM (hsa_circ_0016760 group). A549 cells transfected by pCDNA3.1 empty vector $(100 \mathrm{nM})$ were set as the Ctrl group. The transfection was performed at $37^{\circ} \mathrm{C}$ for $8 \mathrm{~h}$. FGF5 siRNA (Shanghai GenePharma Co., Ltd.) was commercially obtained. A549 cells wereco-transfected bypCDNA3.1-hsa_circ_0016760 vector and FGF5 siRNA (hsa_circ_0016760 + siFGF5 group).In addition, pCDNA3.1-hsa_circ_0016760 vector and miR-145-5p mimic were both used to co-transfect A549 cells, and served as the hsa_circ_0016760 + miR-145-5p mimic group.

All cells were incubated at $37^{\circ} \mathrm{C}$ and $5 \% \mathrm{CO}_{2}$ for $8 \mathrm{~h}$ after transfection. Subsequently, fresh DMEM containing 10\% FBS was used to culture cells for $48 \mathrm{~h}$. RT-qPCR was used for the transfection efficiency detection.

Cell Counting Kit-8 (CCK-8) assay. The proliferation ability of cells was evaluated by CCK- 8 assay. Cells were harvested and dispersed in DMEM (with 10\% FBS) to a density of $1 \times 10^{5} \mathrm{cells} / \mathrm{ml}$. A total of $100 \mu \mathrm{l}$ of cell suspension was added into 96-well plates with 5 multiple wells. Cells were maintained at $37^{\circ} \mathrm{C}$ and $5 \% \mathrm{CO}_{2}$ for 24,48 and $72 \mathrm{~h}$. CCK- 8 solution $(10 \mu \mathrm{l})$ was added into each well and the cells were incubated for $4 \mathrm{~h}$ at $37^{\circ} \mathrm{C}$. The optical density (OD) value was monitored using a microplate reader (Bio-Tek Instruments, Inc.) at a wavelength of $450 \mathrm{~nm}$.

5-Ethynyl-2'-deoxyuridine (EdU) assay. EdU assays were conducted to assess the proliferation ability of cells using an EdU assay kit (Beijing Solarbio Science \& Technology Co., Ltd.) strictly according to the manufacturer's instructions. Briefly, cells were collected and seeded into 6-well plates with $1 \times 10^{5}$ cells/well. EdU buffer $(50 \mu \mathrm{M})$ was used to incubate cells for $2 \mathrm{~h}$ at $37^{\circ} \mathrm{C}$. Then, $4 \%$ formaldehyde was used to fix cells for $30 \mathrm{~min}$ at $37^{\circ} \mathrm{C}$, and $0.1 \%$ Triton X-100 was used for 20 min of permeabilization at $37^{\circ} \mathrm{C}$. EdU solution was then applied to incubate cells for $20 \mathrm{~min}$ at $37^{\circ} \mathrm{C}$ and Hoechst 33342 $(5 \mu \mathrm{g} / \mathrm{ml}$; Beyotime Institute of Biotechnology) was used to stain the nucleus for $15 \mathrm{~min}$ at $37^{\circ} \mathrm{C}$. Under a fluorescence 
Table I. High hsa_circ_0016760 expression is associated with poor prognosis of NSCLC patients.

\begin{tabular}{|c|c|c|c|c|}
\hline \multirow[b]{2}{*}{ Characteristics } & \multirow[b]{2}{*}{ No. of patients } & \multicolumn{2}{|c|}{ Hsa_circ_0016760 expression } & \multirow[b]{2}{*}{ P-value } \\
\hline & & Low $(<$ median $)$ & High ( $\geq$ median) & \\
\hline Number & 80 & 38 & 42 & \\
\hline Age (years) & & & & 0.504 \\
\hline$<65$ & 39 & 19 & 20 & \\
\hline$\geq 65$ & 41 & 19 & 22 & \\
\hline Sex & & & & 0.420 \\
\hline Female & 38 & 19 & 19 & \\
\hline Male & 42 & 19 & 23 & \\
\hline Smoking & & & & 0.412 \\
\hline Yes & 40 & 18 & 22 & \\
\hline No & 40 & 20 & 20 & \\
\hline Tumor size & & & & 0.039 \\
\hline$\leq 3 \mathrm{~cm}$ & 37 & 22 & 15 & \\
\hline$>3 \mathrm{~cm}$ & 43 & 16 & 27 & \\
\hline Differentiation & & & & 0.513 \\
\hline Well differentiated & 37 & 18 & 19 & \\
\hline Lowly or undifferentiated & 43 & 20 & 23 & \\
\hline T classification & & & & 0.023 \\
\hline $\mathrm{T} 1+\mathrm{T} 2$ & 38 & 23 & 15 & \\
\hline $\mathrm{T} 3+\mathrm{T} 4$ & 42 & 15 & 27 & \\
\hline $\mathrm{N}$ classification & & & & 0.037 \\
\hline $\mathrm{N} 0+\mathrm{N} 1$ & 39 & 23 & 16 & \\
\hline $\mathrm{N} 2+\mathrm{N} 3$ & 41 & 15 & 26 & \\
\hline Distant metastasis & & & & 0.251 \\
\hline M1 & 40 & 17 & 23 & \\
\hline M0 & 40 & 21 & 19 & \\
\hline Clinical stage & & & & 0.013 \\
\hline I-II & 39 & 24 & 15 & \\
\hline III-IV & 41 & 14 & 27 & \\
\hline
\end{tabular}

NSCLC, non-small cell lung cancer.

microscope, the EdU-positive cells were photographed and counted as previously described (11).

Transwell assays. Cells were collected and dispersed in serum-free DMEM. The Transwell inserts $(8 \mu \mathrm{m}$ pore size; Corning, Inc.) were inserted into 6-well plates and Matrigel was pre-coated on the upper chamber. Then $5 \times 10^{4}$ cells dispersed in $300 \mu \mathrm{l}$ of serum-free DMEM was added into the upper chamber, while $600 \mu 1$ of DMEM (with $10 \%$ FBS) was added into the lower chamber. Cells were incubated at $37^{\circ} \mathrm{C}, 5 \% \mathrm{CO}_{2}$ for $24 \mathrm{~h}$. The chamber was removed and the non-invading cells were removed with a cotton swab. The invasive cells were fixed at room temperature for $20 \mathrm{~min}$ with $4 \%$ paraformaldehyde, after which, $0.1 \%$ crystal violet was used to stain the cells for $10 \mathrm{~min}$ at room temperature. The number of invasive cells was counted under a confocal microscope in five random fields (magnification, $\mathrm{x} 200$ ). Cell migration was performed according to the aforementioned steps, without the use of Matrigel coated on the upper chamber.

Luciferase reporter gene assay. Through biological software online analysis [Circular RNA Interactome (http://circinteractome.nia.nih.gov/), miRDB (http://www.mirdb.org/) and TargetScan (http://www.targetscan.org/vert_71/)], it was revealed that hsa_circ_0016760 and FGF5 both had binding sites for miR-145-5p. Therefore, H1299 cells were used for the luciferase reporter gene assay to detect the relationship between miR-145-5p and hsa_circ_0016760 (or FGF5). Fragments of hsa_circ_0016760 wild-type (WT) and mutant type (Mut) as well as FGF5 WT and Mut were designed and synthesized by Shanghai GenePharma Co., Ltd.. These fragments were loaded into the pmirGLO luciferase vectors. H1299 cells transfected by miR-145-5p mimic (miR-145-5p mimic group, sequence: 5'-GUCCAGUUUUCCCAGGAAUCCCU-3') and mimic negative control (miR-NC group, sequence: 5'-CGC 
GAGUUAACGGACCAUACGGU-3') were seeded in 6-well plates with serum-free DMEM. Then these cells underwent co-transfection with pmirGLO-hsa_circ_0016760-WT luciferase vector, or pmirGLO-hsa_circ_0016760-Mut luciferase vector, or pmirGLO-FGF5-WT luciferase vector or pmirGLO-FGF5-Mut luciferase vector. Lipofectamine 2000 reagent (Thermo Fisher Scientific, Inc.) was used for the transfection. After $8 \mathrm{~h}$, the serum-free DMEM in each well was replaced by DMEM containing $10 \%$ FBS. After $48 \mathrm{~h}$ of culture at $37^{\circ} \mathrm{C}$ and $5 \% \mathrm{CO}_{2}$, the cells were harvested to detect the luciferase activity using Dual-Glo luciferase assay kit (Promega Corporation) strictly according to the manufacturer's instructions. The relative luciferase activity was normalized to Renilla luciferase activity.

RNA immunoprecipitation (RIP) assay. EZ-Magna RIP kit (EMD Millipore) was used for RIP assays strictly according to the manufacturer's instructions. H1299 cells transfected by miR-145-5p mimic (miR-145-5p mimic group) and mimic negative control (miR-NC group) was harvested and lysed in RIP lysis buffer. Then $100 \mu 1$ of the cell extract was collected for 6-h incubation at $4^{\circ} \mathrm{C}$ with RIP buffer containing magnetic beads conjugated with antibodies against AGO2 (1:200; product code ab5072) or IgG (1:200; product code ab109489; both from Abcam). Wash buffer was used to wash the beads. Subsequently, Proteinase K was applied to incubate the complexes for $30 \mathrm{~min}$ at $55^{\circ} \mathrm{C}$. Finally, the immunoprecipitated hsa_circ_0016760 was purified using phenol, chloroform, salt solution I, salt solution II, precipitate enhancer and absolute ethanol (no RNase), and was assessed with RT-qPCR.

Animal xenograft experiment. Animal experiments involved in the present study were approved by the Animal Ethics Committee of the Affiliated Changzhou No. 2 People's Hospital of Nanjing Medical University. Nude mice $(n=12$; male, 4 weeks old, 18-20 g) were commercially provided by Shanghai Experimental Animal Center, Chinese Academy of Sciences. Mice were kept in a 12-h day/night cycle room with free access to water and food. H1299 cells were transfected by hsa_circ_0016760 shRNA $(100 \mathrm{nM})$ and corresponding negative control (100 nM) (Shanghai GenePharma Co., Ltd.). After $48 \mathrm{~h}$ of transfection, H1299 cells were harvested and washed with phosphate-buffered saline (PBS). A total of $1 \times 10^{6}$ cells dispersed in $100 \mu \mathrm{l}$ PBS was prepared for subcutaneous implantation. Thereafter, 6 nude mice were randomly selected and subcutaneously injected with H1299 cells transfected by hsa_circ_0016760 shRNA. These mice were named the sh-hsa_circ_0016760 group. In addition, the other 6 mice were subcutaneously implanted with H1299 cells transfected by corresponding negative control, which were used as sh-Ctrl group. Mice were maintained for 28 days with free access to food and water. Every 7 days, the longitudinal diameter (L) and latitudinal diameter (D) were measured using a Vernier caliper. The tumor volume was calculated by the equation of $\mathrm{V}=0.5 \times \mathrm{L} \times \mathrm{D}^{2}$. On the 28th day, all mice were sacrificed by rapid neck dislocation. The xenograft tumors were stripped, weighed and then stored at $-80^{\circ} \mathrm{C}$.

Immunohistochemical staining. The xenograft tumors were fixed with $4 \%$ paraformaldehyde for $12 \mathrm{~h}$ at $4^{\circ} \mathrm{C}$, embedded in paraffin and prepared into paraffin tissue sections with a thickness of $4 \mu \mathrm{m}$. The sections were then dewaxed and rehydrated. Sodium citrate buffer was used for the antigen retrieval of the sections. Bovine serum albumin (5\%; Beijing Solarbio Science \& Technology Co., Ltd.) was applied to block the sections for $30 \mathrm{~min}$ at room temperature. Thereafter, the sections were incubated with rabbit anti-Ki67 (product no. 9449; Cell Signaling Technology, Inc.) for $12 \mathrm{~h}$ at $4^{\circ} \mathrm{C}$, and then with goat anti-rabbit HRP conjugated secondary antibody (product no. 7074; Cell Signaling Technology, Inc.) for $1 \mathrm{~h}$ at room temperature. Diaminobenzidine (DAB) and hematoxylin were used for the staining of the sections for $15 \mathrm{~min}$ at room temperature. Under a confocal microscope, the Ki67-positive signals (brown particles) were observed (magnification, x200).

$R T-q P C R$. TRIzol reagent (Takara Bio, Inc.) was used to isolate total RNA in tissues and cells. According to the manufacturer's instructions, cDNA was reverse transcribed using PrimeScript RT reagent Kit (Takara Bio, Inc.). qPCR was performed in a $10-\mu 1$ reaction system, including $2.0 \mu \mathrm{l}$ of cDNA, $0.5 \mu \mathrm{l}$ of forward primer, $0.5 \mu \mathrm{l}$ of reverse primer, $5.0 \mu \mathrm{l}$ of 2X PCR Master mix and $2.0 \mu \mathrm{l}$ of $\mathrm{H}_{2} \mathrm{O}$. The qPCR reaction was performed with SYBR Green reagent (Takara Bio, Inc.) using the ABI 7500 Fast Real-Time PCR System (Applied Biosystems; Thermo Fisher Scientific, Inc.) according to the following conditions: $10 \mathrm{~min}$ initial denaturation at $95^{\circ} \mathrm{C}$, 40 cycles of amplification at $95^{\circ} \mathrm{C}$ for $10 \mathrm{sec}$, annealing at $56^{\circ} \mathrm{C}$ for $45 \mathrm{sec}$ and extension at $60^{\circ} \mathrm{C}$ for $60 \mathrm{sec}$. Primers used were as follows: hsa_circ_0016760 forward, 5'-TGC ATTGGTGCTCAGAAGCG-3' and reverse, 5'-TCTGTTCCT GGGTCTGTGTGC-3'; miR-145-5p forward, 5'-GTCCAG TTTTCCCAGGAATCCCT-3' and reverse, 5'-GCTGTCAAC ATACGCTACGTAACG-3'; FGF5 forward, 5'-CCCGGATGG CAAAGTCAATGG-3' and reverse, 5'-TTCAGGGCAACA TACCACTCCCG-3'; GAPDH forward, 5'-AATGTGTCC GTCGTGGATCTG-3' and reverse, 5'-CAACCTGGTCCT CAGTGTAGC-3'. GAPDH was set as the internal control and the relative expression of hsa_circ_0016760, miR-145-5p and FGF5 mRNA was calculated by $2^{-\Delta \Delta C q}$ method (12).

Western blotting. Total proteins in cells were extracted with RIPA lysis buffer (Beyotime Institute of Biotechnology). Protease inhibitors (Roche Diagnostics) were contained in the RIPA lysis buffer. A BCA Protein Assay kit (Beyotime Institute of Biotechnology) was applied for the determination of total protein concentration. Then $10 \mu \mathrm{l}$ of total protein $(50 \mu \mathrm{g})$ extract was subjected to separation with $10 \%$ sodium dodecyl sulphate-polyacrylamide gel electrophoresis (SDS-PAGE). The separated proteins were transferred onto polyvinylidene fluoride (PVDF) membranes. Subsequently, the membranes were blocked for $1 \mathrm{~h}$ with $5 \%$ non-fat milk at room temperature. Rabbit polyclonal FGF5 antibody (1:1,000; cat. sc-376264; Santa Cruz Biotechnology, Inc.) was used for the incubation of the membrane for $12 \mathrm{~h}$ at $4^{\circ} \mathrm{C}$. Thereafter, goat anti-rabbit secondary antibody (1:5,000; cat. no. PAB10821; Abnova) was applied to incubate the membrane for $1 \mathrm{~h}$ at room temperature. The blots were captured by an enhanced chemiluminescence (ECL) system (Pierce Biotechnology; Thermo Fisher Scientific, Inc.). The intensity of the blots was quantified by 
A
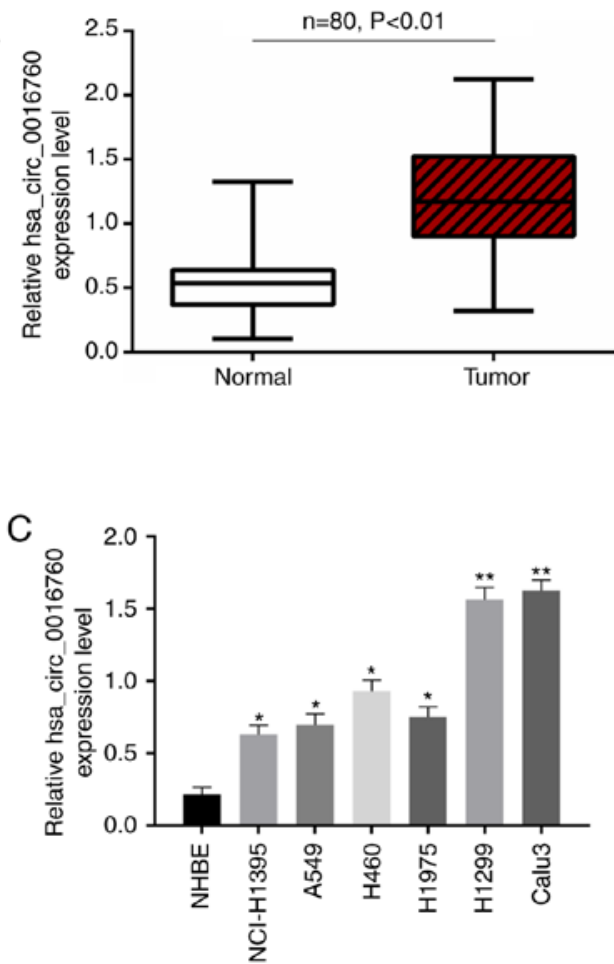

B
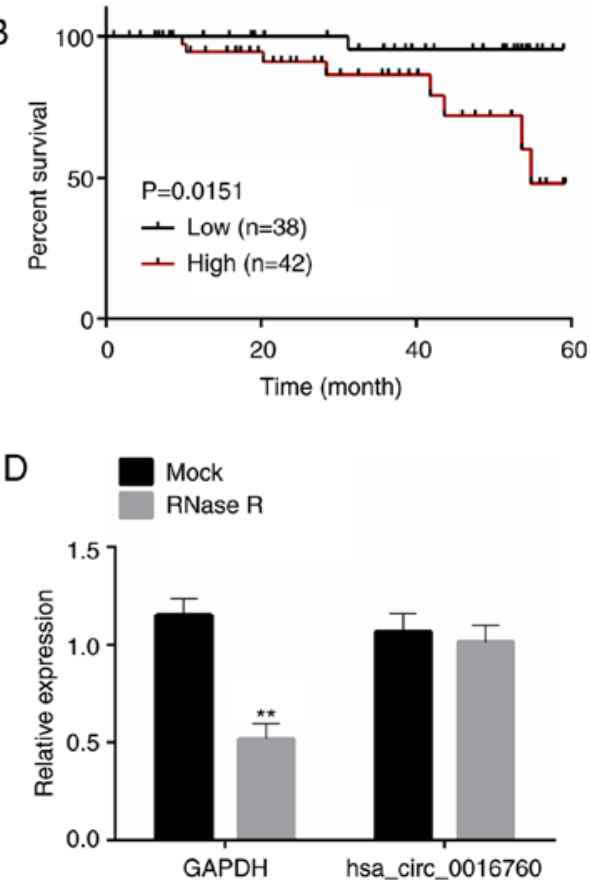

Figure 1.Hsa_circ_0016760 is aberrantly upregulated in NSCLC. (A) RT-qPCR revealed that hsa_circ_0016760 expression was significantly increased in tumor tissues than that in paired adjacent normal tissues. (B) Kaplan-Meier survival analysis indicated that high hsa_circ_0016760 expression was significantly associated with poor 60-month survival of NSCLC patients. (C) The expression of hsa_circ_0016760 was significantly upregulated in the 6 NSCLC cell lines than that in NHBE cells. ${ }^{*} \mathrm{P}<0.05$ and ${ }^{* *} \mathrm{P}<0.01$ when compared with hsa_circ_0016760 expression in NHBE cells. (D) Hsa_circ_0016760 could resist the degradation of RNase R, indicating that hsa_circ_0016760 possessed a stable circular structure and could be stably expressed in NSCLC. ${ }^{* *} \mathrm{P}<0.01$ when compared with the Mock group. NSCLC, non-small cell lung cancer.

ImageJ software (version 1.51t; National Institutes of Health). The internal control was GAPDH in this research.

Statistical analysis. The statistical analysis was processed through SPSS 19.0 software (IBM Corp.) The graphs were constructed using GraphPad Prism 6 software (GraphPad Software, Inc.). Two-tailed paired Student's t-test and one-way analysis of variance (ANOVA) was respectively used for the comparison between two groups and more than two groups. Tukey's post hoc test was used to validate ANOVA for pairwise comparisons. The relationship between hsa_circ_0016760 expression and the clinicopathological characteristics of NSCLC patients was analyzed by $\chi^{2}$ test. Kaplan-Meier survival analysis and log-rank tests were applied for the analysis of survival curves. Among hsa_circ_0016760,miR-145-5p and FGF5, Pearson's correlation analysis was used to assess the correlation between two genes. $\mathrm{P}<0.05$ was considered to indicate a statistically significant difference.

\section{Results}

Hsa_circ_0016760 is aberrantly upregulated in NSCLC, which is associated with the poor prognosis of patients. The present study firstly explored the expression of hsa_circ_0016760 in 80 pairs of tumor tissues/adjacent normal tissues. The results presented in Fig. 1A revealed thathsa_circ_0016760 expression was significantly increased in tumor tissues than that in paired adjacent normal tissues $(\mathrm{P}<0.01)$. Thereafter, the correlation between hsa_circ_0016760 expression and the clinicopathological characteristics of NSCLC patients was analyzed to evaluate the significance of hsa_circ_0016760 upregulation in NSCLC. As a result, high hsa_circ_0016760 expression was significantly associated with poor 60 -month survival $(\mathrm{P}=0.0151)$, large tumor size $(\mathrm{P}=0.039)$, advanced $\mathrm{T}$ classification $(\mathrm{P}=0.023), \mathrm{N}$ classification $(\mathrm{P}=0.037)$ and clinical stage $(\mathrm{P}=0.013)$ (Fig. 1B; Table I). The expression of hsa_circ_0016760 in NHBE cells and 6 NSCLC cell lines (NCL-H1395, A549, H460, H1975, H1299 and Calu3) was further assessed. Notably, the 6 NSCLC cell lines were expressed significantly higher hsa_circ_0016760 than the NHBE cells $(\mathrm{P}<0.05$ and $\mathrm{P}<0.01)$. Among the 6 NSCLC cell lines, H1299 and Calu3 cell lines had higher hsa_circ_0016760 expression than the other 4 NSCLC cell lines. Therefore, H1299 and Calu3 cell lines were used in subsequent experiments (Fig. 1C). To demonstrate the expression stability of hsa_circ_0016760, total RNA in tumor tissues of NSCLC patients was collected and incubated with RNase R. As a result, RNase R treatment significantly decreased GAPDH expression $(\mathrm{P}<0.01)$, but had no obvious effect on the expression level of hsa_circ_0016760 (Fig. 1D). Thus, hsa_circ_0016760 possessed as table circular structure, which could be stably expressed in NSCLC. Collectively it was revealed that the high expression of hsa_circ_0016760 in NSCLC was associated with the poor prognosis of patients.

Hsa_circ_0016760 silencing suppresses NSCLC cell proliferation, migration and invasion in vitro. H1299 and Calu3 cells were transfected by hsa_circ_0016760 siRNA. 

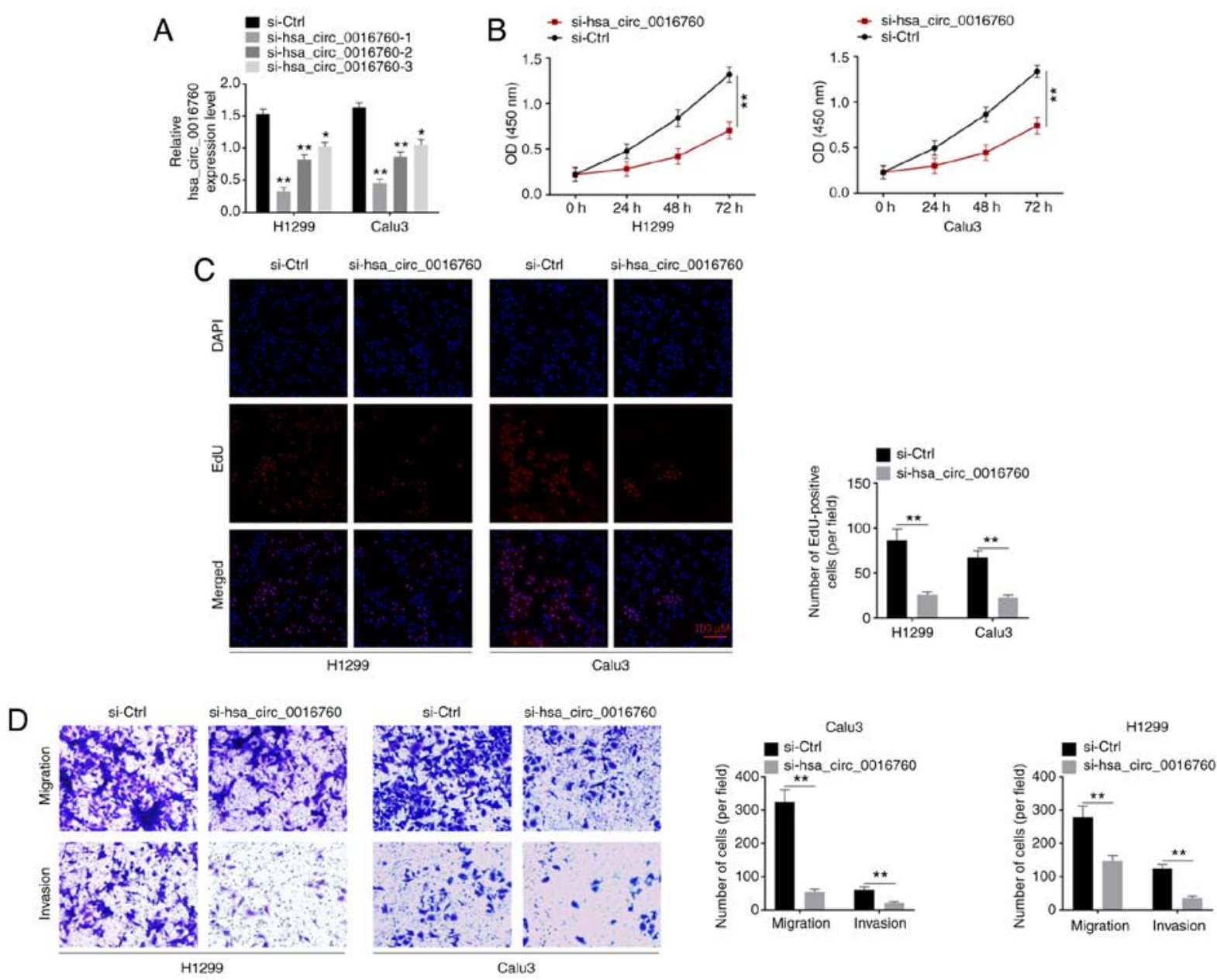

Figure 2. Hsa_circ_0016760 silencing suppresses NSCLC cell proliferation, migration and invasion in vitro. (A) RT-qPCR revealed that hsa_circ_0016760 expression in H1299 and Calu3 cells was successfully silenced via transfection. (B) CCK-8 assays revealed that hsa_circ_0016760 silencing significantly reduced H1299 and Calu3 cell proliferation. (C) An EdU assay revealed that hsa_circ_0016760 silencing significantly decreased the number of EdU-positive cells. 1Bar=50 $\mu \mathrm{m}$. (D) Transwell assays revealed that hsa_circ_0016760 silencing significantly decreased the number of migrated and invasive H1299 and Calu3 cells. ${ }^{*} \mathrm{P}<0.05$ and ${ }^{* *} \mathrm{P}<0.01$ when compared with the si-Ctrl group. NSCLC, non-small cell lung cancer; RT-qPCR, reverse transcription-quantitative polymerase chain reaction; CCK-8, Cell Counting Kit-8; EdU, 5-ethynyl-2'-deoxyuridine; si-, small interfering; Ctrl, control.

RT-qPCR was performed to monitor the transfection efficiency. As revealed in Fig. 2A, the si-hsa_circ_0016760-1, si-hsa_ circ_0016760-2 and si-hsa_circ_0016760-3 groups of H1299 and Calu3 cells exhibited significantly lower hsa_circ_0016760 expression than that of the si-Ctrl group $(\mathrm{P}<0.05$ and $\mathrm{P}<0.01)$. Thus, hsa_circ_0016760 expression in H1299 and Calu3 cells was successfully silenced via transfection. Notably, the si-hsa_ circ_0016760-1 group exhibited lower hsa_circ_0016760 expression than that of the si-hsa_circ_0016760-2 group and si-hsa_circ_0016760-3 group of H1299 and Calu3 cells. Therefore, the si-hsa_circ_0016760-1 group of H1299 and Calu3 cells was used in subsequent experiments, and was renamed as si-hsa_circ_0016760 in the following studies.

Next, the phenotype of H1299 and Calu3 cells was explored to research the effect of hsa_circ_0016760 on NSCLC development in vitro. The proliferation of H1299 and Calu3 cells was analyzed by CCK-8 and EdU assays respectively. The results revealed that the si-hsa_circ_0016760 group of H1299 and Calu3 cells had a significantly lower OD value than that of the si-Ctrl group $(\mathrm{P}<0.01)$ (Fig. 2B). In addition, a significantly less EdU-positive number of cells was observed in the si-hsa_circ_0016760 group when compared with si-Ctrl group of H1299 and Calu3 cells ( $\mathrm{P}<0.01)$ (Fig. 2C). In addition, cell migration and invasion abilities were investigated by Transwell assay. In comparison with the siCtrl group, the number of migrated and invasive cells of the si-hsa_circ_0016760 group of $\mathrm{H} 1299$ and Calu3 cells was significantly decreased $(\mathrm{P}<0.01)$ (Fig. 2D). These data revealed that hsa_circ_0016760 silencing suppressed NSCLC cell proliferation, migration and invasion in vitro.

Hsa_circ_0016760 facilitates FGF5 expression via sponging miR-145-5p. The transfection efficiency of H1299 and Calu3 cells was assessed by RT-qPCR. Compared with the miR-NC group, the miR-145-5p mimic group of H1299 and Calu3 cells had significantly increased miR-145-5p expression $(\mathrm{P}<0.01)$ (Fig. 3A). Thus, H1299 and Calu3 cells were successfully transfected by miR-145-5p mimic and mimic NC. The exact target of hsa_circ_0016760 was investigated in order to elucidate the mechanism of hsa_circ_0016760 in the promotion of the malignant phenotype of NSCLC cells. According to online bioinformatics databases (Circular RNA Interactome and miRDB), miR-145-5p may be a target of hsa_circ_0016760, as it contained a binding site for 


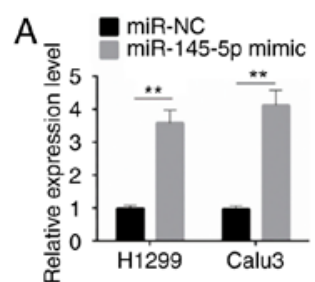

B hsa_circ_0016760 5' ACAGAUUGCUGACAGAACUGGAA 3 III | IIIII miR-145-5p 3' UCCCUAAGGACCCUUUUGACCUG 5
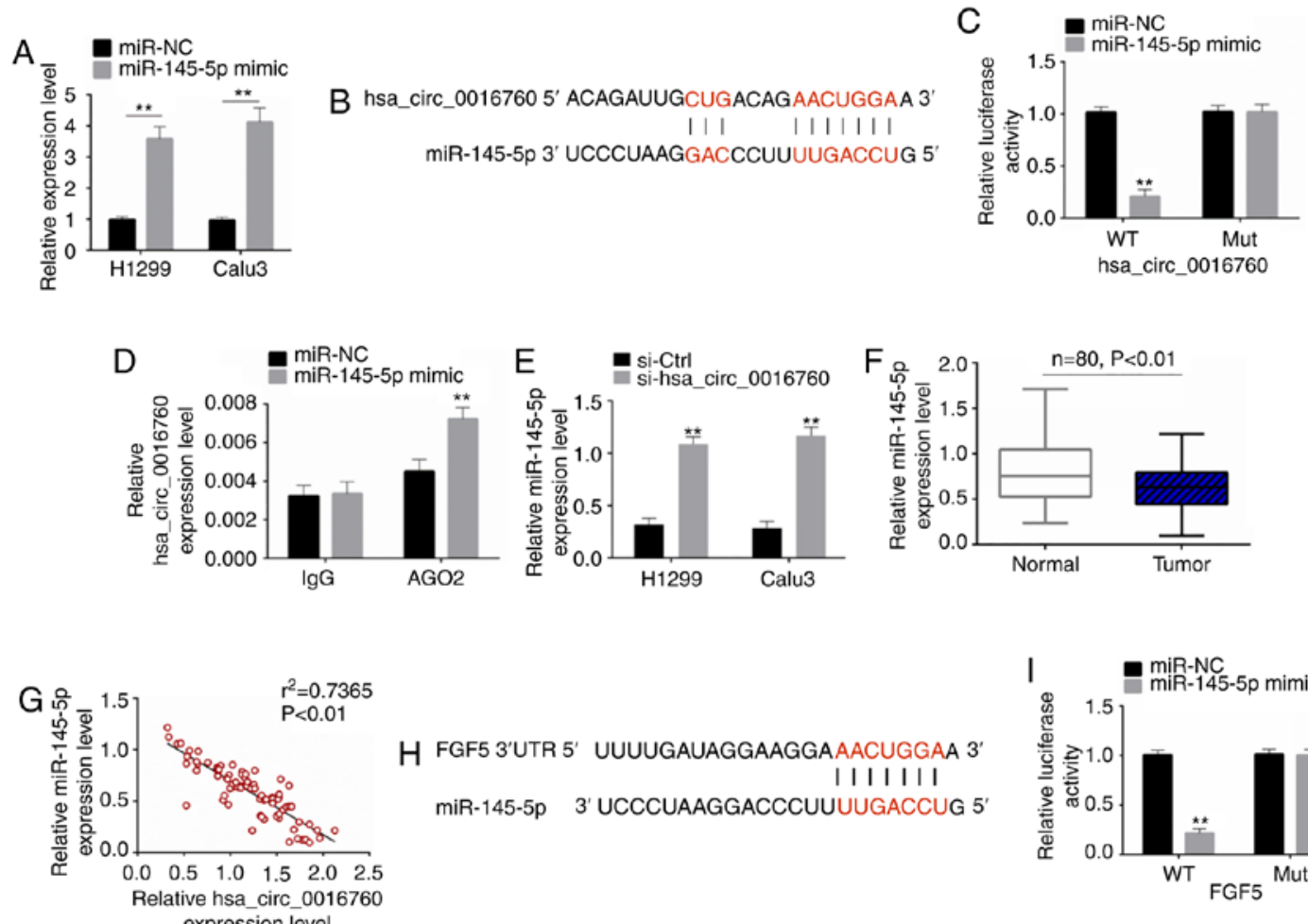
H FGF5 3'UTR 5' UUUUGAUAGGAAGGAAACUGGAA 3' | | | | | | miR-145-5p 3' UCCCUAAGGACCCUUUUGACCUG 5'

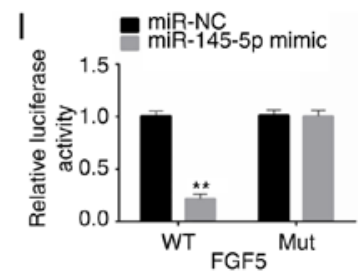
expression level
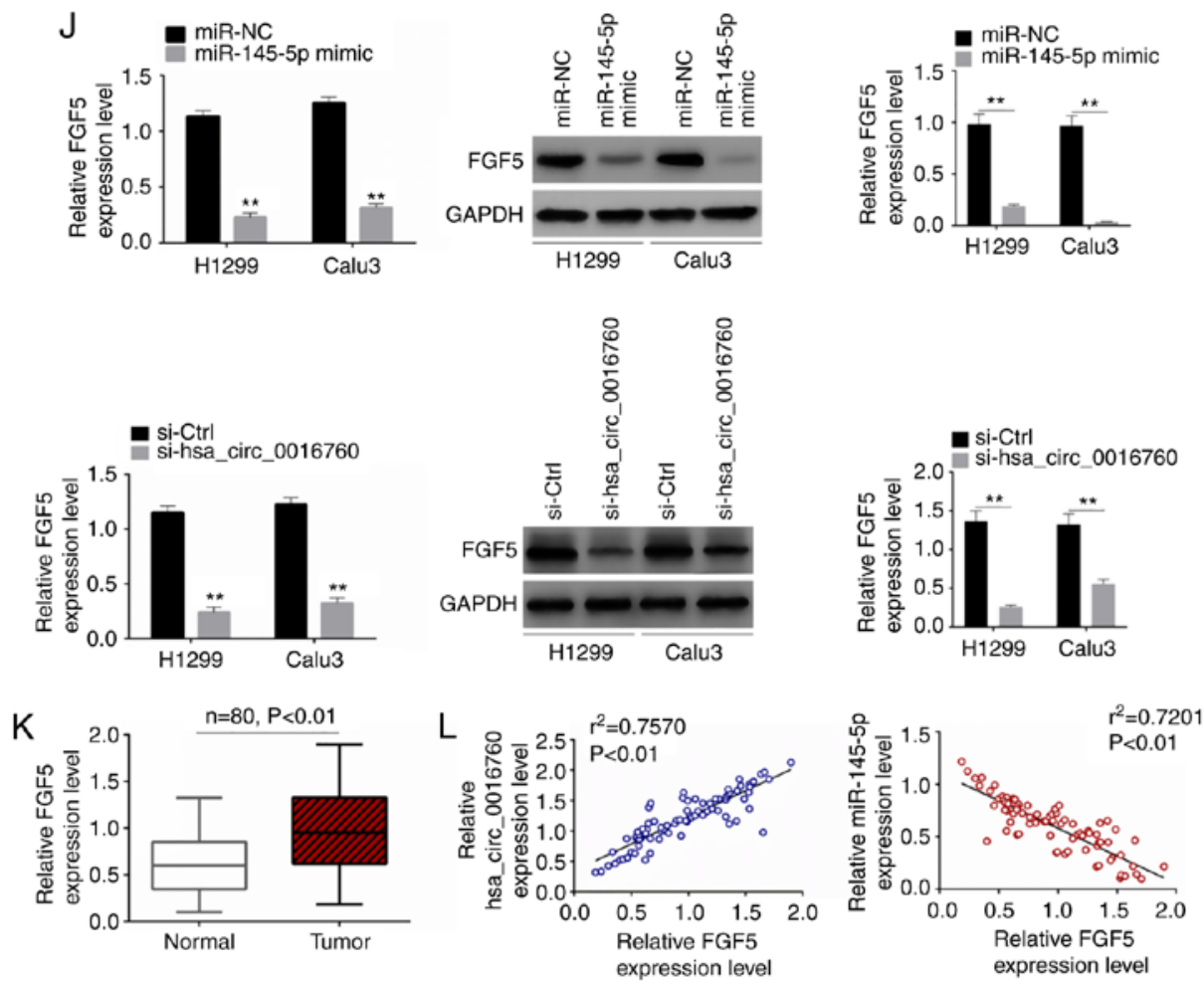

Figure 3.Hsa_circ_0016760 facilitates FGF5 expression via sponging miR-145-5p. (A) RT-qPCR revealed that H1299 and Calu3 cells were successfully transfected by miR-145-5p mimic and mimic NC. ${ }^{* *} \mathrm{P}<0.01$ when compared with miR-NC group. (B) According to an online bioinformatics database, miR-145-5p contained a binding site for hsa_circ_0016760. (C) A luciferase reporter gene assay indicated that miR-145-5p was a target of hsa_circ_0016760. ${ }^{* *} \mathrm{P}<0.01$ when compared with miR-NC group. (D) A RIP assay revealed that, hsa_circ_0016760 could be effectively precipitated by miR-145-5p, and miR-145-5p was further confirmed to be a target of hsa_circ_0016760. ${ }^{* *} \mathrm{P}<0.01$ when compared with miR-NC group. (E) Hsa_circ_0016760 silencing significantly increased the expression of miR-145-5p in $\mathrm{H} 1299$ and Calu3 cells. ${ }^{* *} \mathrm{P}<0.01$ when compared with the si-Crtl group. (F) miR-145-5p expression was significantly reduced in tumor tissues compared with adjacent normal tissues. (G) miR-145-5p expression was negatively correlated with hsa_circ_0016760 in NSCLC tumor tissues. (H) According to TargetScan online database, FGF5 maybe target of miR-145-5p, since it possessed a binding site for miR-145-5p. (I) A luciferase reporter gene assay revealed that FGF5 was a target of miR-145-5p. ${ }^{* *} \mathrm{P}<0.01$ when compared with miR-NC group. (J) In H1299 and Calu3 cells, miR-145-5p upregulation significantly reduced FGF5 mRNA and protein expression. In addition, hsa_circ_0016760 downregulation significantly reduced FGF5 mRNA and protein expression. ${ }^{* * *} \mathrm{P}<0.01$ when compared with miR-NC group or si-Crtl group. (K) FGF5 expression was significantly increased in tumor tissues compared with adjacent normal tissues. (L) Pearson's correlation analysis indicated that, in NSCLC tumor tissues, the expression level of FGF5was positively correlated with the expression level of hsa_circ_0016760. Conversely, a negative correlation was revealed between the expression levels ofFGF5 and miR-145-5p. NSCLC, non-small cell lung cancer; RT-qPCR, reverse transcription-quantitative polymerase chain reaction; NC, negative control; si-, small interfering; Ctrl, control; RIP, RNA immunoprecipitation. 
hsa_circ_0016760 (Fig. 3B). Luciferase reporter gene assay and RIP assay were then performed to verify the relationship between hsa_circ_0016760 and miR-145-5p. As revealed in Fig. 3C, the miR-145-5p mimic group of H1299 cells exhibited significantly decreased relative luciferase activity of hsa_circ_0016760-WT reporter than the miR-NC group $(\mathrm{P}<0.01)$. However, the difference in relative luciferase activity of hsa_circ_0016760-Mut reporter between the miR-145-5p mimic group and miR-NC group was not statistically significant. Furthermore, based on the results from the RIP assay, it was observed that hsa_circ_0016760 could be effectively precipitated by the AGO2 antibody. Notably, hsa_circ_0016760 expression was significantly increased in the miR-145-5p mimic group when compared with the miR-NC group $(\mathrm{P}<0.01)$ (Fig. 3D). Thus, miR-145-5p was confirmed as a target of hsa_circ_0016760. For the si-hsa_circ_0016760 group, significantly higher miR-145-5p expression was observed compared with the si-Ctrl group of H1299 and Calu3 cells $(\mathrm{P}<0.01)$ (Fig. 3E). In addition, in comparison with adjacent normal tissues, significantly decreased miR-145-5p expression was observed in tumor tissues of NSCLC patients $(\mathrm{P}<0.01)$ (Fig. 3F). In tumor tissues of NSCLC patients, the expression level ofmiR-145-5p was negatively correlated with the expression level of hsa_circ_0016760 ( $\mathrm{P}<0.01)$ (Fig. 3G). Therefore, it was confirmed that miR-145-5p was directly inhibited by hsa_circ_0016760 in NSCLC according to these data.

According to TargetScan online database, FGF5 maybe target of miR-145-5p, since it possessed a binding site for miR-145-5p (Fig. 3H). A luciferase reporter gene assay revealed that the miR-145-5p mimic group exhibited significantly reduced relative luciferase activity of FGF5-WT reporter compared with the miR-NC group of H1299 cells $(\mathrm{P}<0.01)$, whereas no statistically significant difference was revealed in the relative luciferase activity of the FGF5-Mut reporter between the miR-NC group and miR-145-5p mimic group (Fig. 3I). Hence, FGF5 was identified as a target of miR-145-5p. In vitro studies revealed that the miR-145-5p mimic group expressed significantly decreased FGF5 mRNA and protein expression levels than those of the miR-NC group of H1299 and Calu3 cells $(\mathrm{P}<0.01)$. Concurrently, relative to the si-Ctrl group, the FGF5 mRNA and protein expression levels of the si-hsa_circ_0016760 group of H1299 and Calu3 cells were significantly decreased $(\mathrm{P}<0.01)$ (Fig. 3J). Moreover, for NSCLC patients, significantly increased FGF5 expression was observed in tumor tissues than that in adjacent normal tissues $(\mathrm{P}<0.01)$ (Fig. 3K). The correlation analysis of FGF5 and hsa_circ_0016760 (or miR-145-5p) in tumor tissues revealed that, the expression level of FGF5 was positively correlated with hsa_circ_0016760 $(\mathrm{P}<0.01)$. Conversely, a negative correlation was revealed for FGF5 and miR-145-5p expression levels in tumor tissues $(\mathrm{P}<0.01)$ (Fig. 3L). All of these data demonstrated that hsa_circ_0016760 enhanced the expression of FGF5 via sponging miR-145-5p.

Hsa_circ_0016760 exacerbates the malignant development of NSCLC by sponging the miR-145-5p/FGF5 axis. The mechanism of hsa_circ_0016760 in promoting NSCLC malignant development was verified by rescue experiments. A549 cells had relatively low hsa_circ_0016760 expression among the 6 NSCLC cell lines. Thus, hsa_circ_0016760 was overexpressed in A549 cells. As revealed in Fig. 4A, compared with the Ctrl group, FGF5 protein expression in the hsa_circ_0016760 group of A549 cells was significantly increased $(\mathrm{P}<0.01)$. A549 cells were also subjected to co-transfection. The results revealed that compared with the hsa_circ_0016760 group, significantly decreased FGF5 protein expression was observed in the hsa_circ_0016760 + miR-145-5p mimic group and hsa_circ_0016760 + si-FGF5 group of A549 cells $(\mathrm{P}<0.01)$. In addition, the expression of hsa_circ_0016760, miR-145-5p and FGF5 mRNA in A549 cells of the 4 groups was assessed. As revealed in Fig. 4B, compared with the Ctrl group, the hsa_circ_0016760 group exhibited higher hsa_circ_0016760 expression, lower miR-145-5p expression and higher FGF5 mRNA expression in A549 cells $(\mathrm{P}<0.01)$. However, in comparison with the hsa_circ_0016760 group, the hsa_circ_0016760 + miR-145-5p mimic group and hsa_circ_0016760 + si-FGF5 group presented significantly decreased hsa_circ_0016760 expression, increased miR-145-5p expression and decreased FGF5 mRNA expression $(\mathrm{P}<0.01)$.

Then, the malignant phenotype of A549 cells was investigatedin vitro.According toaCCK-8 assay,thehsa_circ_0016760 group of A549 cells exhibited a significantly increased OD value than that of the Ctrl group $(\mathrm{P}<0.01)$. Conversely, compared with the hsa_circ_0016760 group, the OD values of A549 cells in the hsa_circ_0016760 + miR-145-5p mimic group and hsa circ_0016760 + si-FGF5 group were significantly decreased $(\mathrm{P}<0.01)$ (Fig. 4C). Similarly, an EdU assay revealed that, more Edu-positive cells were observed in the hsa_circ_0016760 group when compared with the Ctrl group of A549 cells $(\mathrm{P}<0.01)$. However, the number of Edu-positiveA549 cells in thehsa_circ_0016760 + miR-145-5p mimic group and hsa circ_0016760 + si-FGF5 group were significantly decreased compared with the hsa_circ_0016760 group $(\mathrm{P}<0.01)$ (Fig. 4D). Transwell assays revealed significantly increased migration and invasion abilities of A549 cells in thehsa_circ_0016760 group compared with the Ctrl group $(\mathrm{P}<0.01)$. Conversely, decreased migration and invasion abilities of A549 cells were observed in the hsa_circ_0016760 + miR-145-5p mimic and hsa_circ_0016760 + si-FGF5 groups in comparison with the hsa_circ_0016760 group $(\mathrm{P}<0.01)$ (Fig. 4E). Hence, these results revealed that hsa_circ_0016760 exacerbated the malignant development of NSCLC by targeting the miR-145-5p/FGF5 axis.

Hsa_circ_0016760 silencing inhibits NSCLC cell growth in nude mice. To explore the effect of hsa_circ_0016760 on NSCLC development in vivo, H1299 cells were transfected with hsa_circ_0016760 shRNA and corresponding NC and subcutaneously implanted into nude mice. The results revealed that compared with the sh-NC group, the tumor volume and weight of sh-hsa_circ_0016760 group were significantly reduced $(\mathrm{P}<0.01)$ (Fig. 5A and $\mathrm{B})$. Thus, silencing of hsa_circ_0016760 markedly slowed down the growth of xenograft tumors in vivo. RT-qPCR revealed that, xenograft tumors of sh-hsa_circ_0016760 group exhibited significantly decreased hsa_circ_0016760 expression, increasedmiR-145-5p expression and decreasedFGF5 expression compared with the sh-NC groups $(\mathrm{P}<0.01)$ (Fig. 5C). Immunohistochemical staining revealed decreased Ki67-positive signals in the 
A

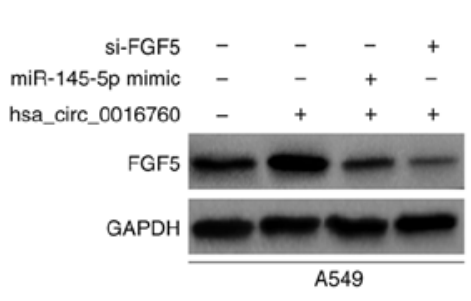

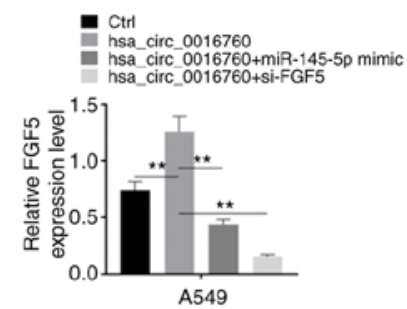
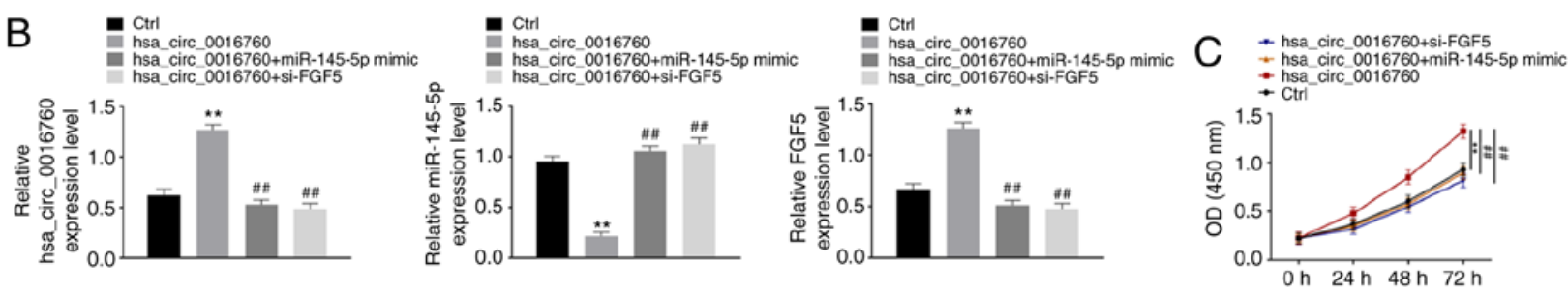

si-FGF5
miR-145-5p mimic
hsa_circ_0016760
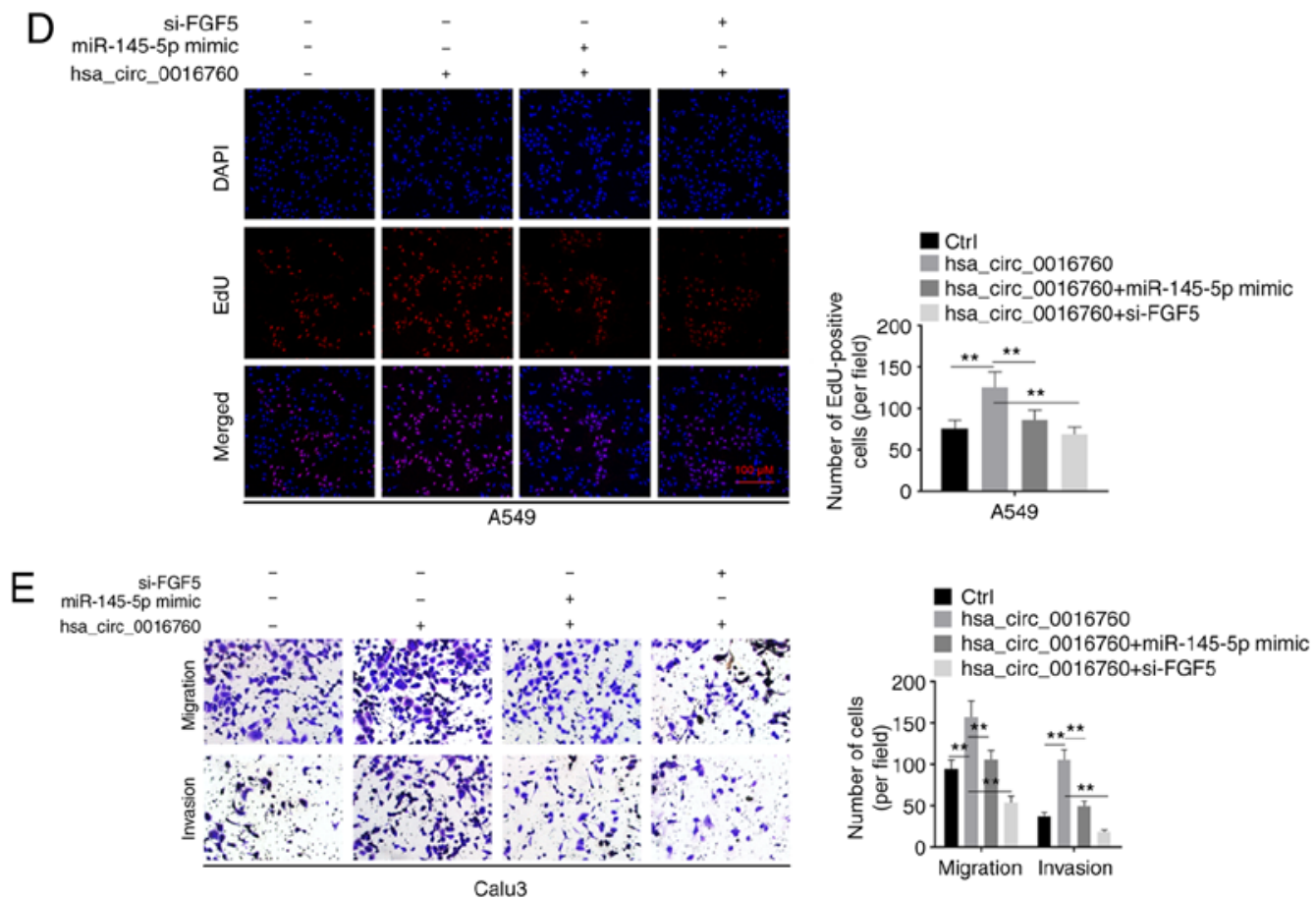

Figure 4. Hsa_circ_0016760 exacerbates the malignant development of NSCLC by targeting the miR-145-5p/FGF5 axis. (A) FGF5 protein expression in A549 cells was successfully regulated by transfection. ${ }^{* *} \mathrm{P}<0.01$. (B) Expression of hsa_circ_0016760, miR-145-5p and FGF5 mRNA in A549 cells was successfully regulated by transfection. ${ }^{* *} \mathrm{P}<0.01$ when compared with the Ctrl group; ${ }^{* \#} \mathrm{P}<0.01$ when compared with the hsa_circ_0016760 group. (C) A CCK-8 assay revealed that miR-145-5p upregulation and FGF5 downregulation reversed the promoting effect of hsa_circ_0016760 on A549 cell proliferation. ${ }^{* *} \mathrm{P}<0.01$ when compared with the Ctrl group; ${ }^{\# \#} \mathrm{P}<0.01$ when compared with the hsa_circ_0016760 group. (D) An EdU assay revealed that miR-145-5p upregulation and FGF5 downregulation weakened the promoting effect of hsa_circ_0016760 on A549 cell proliferation. 1Bar=50 $\mu \mathrm{m}$. ${ }^{* *} \mathrm{P}<0.01$. (E) Transwell assays revealed that miR-145-5p upregulation and FGF5 downregulation reversed the promoting effect of hsa_circ_0016760 on A549 cell migration and invasion abilities. ${ }^{* *} \mathrm{P}<0.01$. NSCLC, non-small cell lung cancer; CCK-8, Cell Counting Kit-8; EdU, 5-ethynyl-2'-deoxyuridine; si-, small interfering; Ctrl, control.

xenograft tumors of the sh-hsa_circ_0016760 group when compared with the sh-NC group (Fig. 5D). Therefore, hsa circ_0016760 silencing inhibited NSCLC cell growth in vivo.

\section{Discussion}

In recent years, the biological role of circRNAs in human tumors has attracted increasing attention. Several functions of circRNAs have been elucidated, including RNA transport, regulation of translation and protein binding (13). Notably, circRNAs display the potential of gene regulation and have been confirmed as efficient sponges for microRNAs (14). Currently, a number of circRNAs have been revealed to participate in the regulation of NSCLC by indirectly regulating coding gene expression via sponging microRNAs (15-17). The discovery of these circRNAs and related molecular mechanisms in regulating NSCLC progression provides wide selection for the targeted treatment of patients. The discovery of more circRNAs is clinically significant for the targeted treatment of NSCLC patients. In the present research, hsa_circ_0016760 was identified as a novel target for NSCLC, as well as an oncogene of NSCLC and was associated with poor prognosis of patients. In terms of the mechanism, it was reported that hsa_circ_0016760 exacerbated the malignant development of NSCLC by enhancing FGF5 expression via sponging of miR-145-5p. 

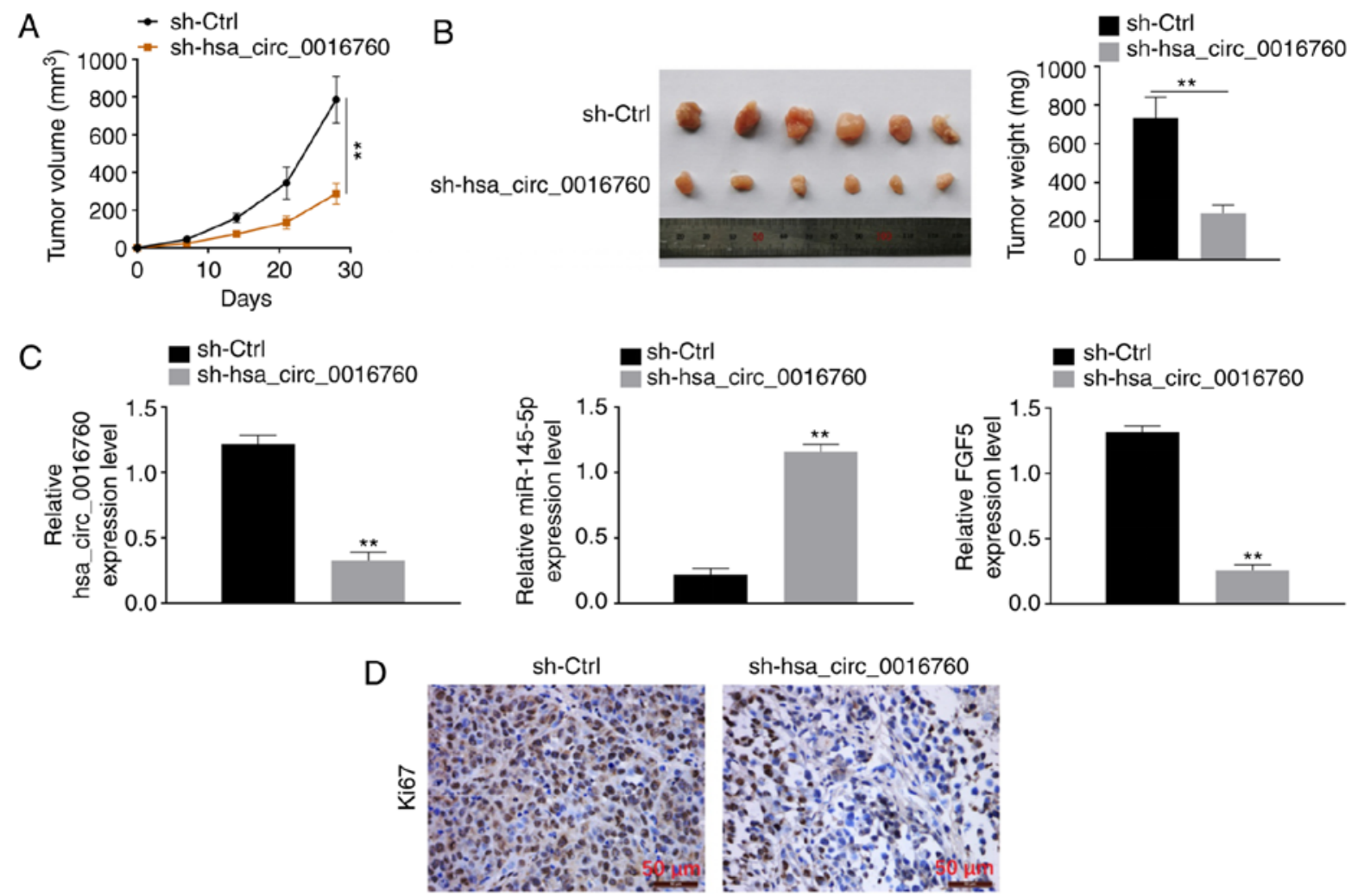

Figure 5. Hsa_circ_0016760 silencing inhibits NSCLC cell growth in nude mice. (A) Silencing of hsa_circ_0016760 significantly reduced the volume of xenograft tumors in vivo. ${ }^{* *} \mathrm{P}<0.01$. (B) Silencing of hsa_circ_0016760 significantly decreased the weight of xenograft tumors in vivo. ${ }^{* *} \mathrm{P}<0.01$. (C) In xenograft tumors, significantly decreased hsa_circ_0016760 expression, increased miR-145-5p expression and decreased FGF5 expression was observed after hsa_circ_0016760 silencing. ${ }^{* *} \mathrm{P}<0.01$ when compared with the sh-NC group. (D) Immunohistochemical staining revealed that silencing of hsa_circ_0016760 significantly reduced Ki67 expression in xenograft tumors. 1Bar=50 $\mu \mathrm{m}$. NSCLC, non-small cell lung cancer; sh-, short hairpin; Ctrl, control.

In the present study, miR-145-5p was identified as a tumor suppressor gene of NSCLC. Gan et al (18) revealed that miR-145-5p had clinical value in the diagnosis and treatment of NSCLC. Through meta-analysis and microRNA microarray analysis, it was determined that, miR-145-5p expression was significantly decreased in NSCLC tissues compared with that in adjacent non-tumor tissues and decreased miR-145-5p expression was closely related to the positive lymph node metastasis of NSCLC patients. Epithelial-mesenchymal transition (EMT) is involved in the important biological process of tumor migration and metastasis. Chang et al (8) demonstrated that miR-145-5p could suppress the EMT in NSCLC by inhibiting the activity of the c-Jun N-terminal kinase (JNK) signaling pathway via targeting mitogen-activated protein kinase kinase kinase 1 (MAP3K1). Results from the present study also identified that, as a tumor suppressor gene, miR-145-5p expression was reduced in NSCLC samples. According to existing literature, miR-145-5p in NSCLC has been reported to be regulated by multiple long-chain non-coding RNAs, including SNHG1, JPX and PVT1. These long-chain non-coding RNAs have been revealed to exhibit cancer-promoting effects in NSCLC by enhancing the expression of downstream coding genes via sponging miR-145-5p (19-21). At present, studies on whether miR-145-5p is regulated by circRNAs in NSCLC are scarce. The present study reported for the first time, to the best of our knowledge, that miR-145-5p was sponged by hsa_circ_0016760 in NSCLC.
In the present study, FGF5 expression was revealed to be upregulated in NSCLC and FGF5 was demonstrated as a potential target gene of miR-145-5p. After overexpression of miR-145-5p, the expression of FGF5 mRNA and protein levels were both decreased. Thus, miR-145-5p may suppress FGF5 expression at transcription and translation levels. Rescue experiments revealed that silencing of FGF5 reversed the promoting effect of hsa_circ_0016760 on NSCLC cell malignant phenotype, including proliferation, migration and invasion. Thus, FGF5 was an oncogene in NSCLC. Zhao et al (9) suggested that FGF5 was overexpressed in lung adenocarcinoma. High FGF5 expression was an independent prognostic factor for patients, and was associated with worse overall survival and relapse-free survival in lung adenocarcinoma patients. Zhou et al (22) revealed that FGF5 expression was aberrantly increased in NSCLC tissues and cell lines. The inhibition of FGF5 significantly suppressed NSCLC cell line proliferation, migration and invasion. It is suggested that FGF5 may be a promising treatment strategy for NSCLC. Furthermore, overexpression of FGF5 has also been revealed to contribute to malignant development in several other human tumors, such as osteosarcoma, melanoma and breast cancer (23-25). Similarly, in the present study, FGF5 played a role in the promotion of the malignant development of NSCLC.

There is a limitation in the present study. It was observed that miR-145-5p overexpression reduced the expression levels of FGF5 mRNA and protein. It was speculated that miR-145-5p may suppress FGF5 expression at both the transcription and 
translation levels. However, we could not conduct more experiments to verify this theory. This point will be the focus of our future research.

In summary, the present study reported the role and mechanism of hsa_circ_0016760 in regulating NSCLC progression. It was demonstrated that hsa_circ_0016760 was an oncogene in NSCLC, and was related to the poor prognosis of patients. Hsa_circ_0016760 silencing could inhibit the malignant progression of NSCLC in vitro and in vivo. With regard to the mechanism, hsa_circ_0016760 exacerbated the malignant development of NSCLC by enhancing FGF5 expression via sponging miR-145-5p. Therefore, hsa_circ_0016760 was recommended as a novel potential target for the treatment of NSCLC.

\section{Acknowledgements}

Not applicable.

\section{Funding}

The present study was supported by the Major Science and Technology Project of Changzhou Health Commission (grant no. ZD201910), the 'Six One Project' Research Projects of High-level Medical Personnel of Jiangsu Province (grant no. LGY2019025), the Medical Scientific Research Foundation of Jiangsu Commission of Health (grant no. H2018083), the High-level Talent Selection and Training Project of The 16th Batch of 'Six Talent Peak' of Jiangsu Province (grant no. WSN-245), the 333 High-Level Talent Training Project (grant no. 2016, III-0719), the High-Level Medical Talents Training Project (grant no. 2016CZBJ042) and the Jiangsu Provincial Medical Youth Talent [Jiangsu Health Scientific Education (2017) No. 3].

\section{Availability of data and materials}

The datasets used during the present study are available from the corresponding author upon reasonable request.

\section{Authors' contributions}

ZZ, KY and QW designed the study. ZZ, QW, MZ, and JT performed the experiments. BZ and KY performed the data analysis. ZZ and KY wrote the manuscript. All authors read and approved the manuscript and agree to be accountable for all aspects of the research in ensuring that the accuracy or integrity of any part of the work are appropriately investigated and resolved.

\section{Ethics approval and consent to participate}

The present study was approved (approval no. H3511EC) by the Ethics Committee of the Affiliated Changzhou No. 2 People's Hospital of Nanjing Medical University (Changzhou, China). All patients volunteered to join the study and written informed consent was signed by all subjects. Animal experiments involved in the present study were approved (approval no. 20140351AEC) by the Animal Ethics Committee of the Affiliated Changzhou No. 2 People's Hospital of Nanjing Medical University.

\section{Patient consent for publication}

Not applicable.

\section{Competing interests}

The authors declare that they have no competing interests.

\section{References}

1. Brahmer JR, Govindan R, Anders RA, Antonia SJ, Sagorsky S, Davies MJ, Dubinett SM, Ferris A, Gandhi L, Garon EB, et al: The society for immunotherapy of cancer consensus statement on immunotherapy for the treatment of non-small cell lung cancer (NSCLC). J Immunother Cancer 6: 75, 2018.

2. He W, Zhang H, Wang Y, Zhou Y, Luo Y, Cui Y, Jiang N, Jiang W, Wang H, Xu D, et al: CTHRC1 induces non-small cell lung cancer (NSCLC) invasion through upregulating MMP-7/MMP-9. BMC Cancer 18: 400, 2018.

3. Chen S, Shi F, Zhang W, Zhou Y and Huang J: miR-744-5p inhibits non-small cell lung cancer proliferation and invasion by directly targeting PAX2. Technol Cancer Res Treat 18: $1533033819876913,2019$.

4. Nicolaou A, Northoff BH, Zhao Z, Kohlmaier A, Sass K, Rose-John S, Steffens S, Weber C, Teupser D and Holdt LM: The ADAM17 metalloproteinase maintains arterial elasticity. Thromb Haemost 118: 210-213, 2018.

5. Zhang J, Hou L, Liang R, Chen X, Zhang R, Chen W and Zhu J: CircDLST promotes the tumorigenesis and metastasis of gastric cancer by sponging miR-502-5p and activating the NRAS/MEK1/ERK1/2 signaling. Mol Cancer 18: 80, 2019.

6. Zhang S, Zeng X, Ding T, Guo L, Li Y, Ou S and Yuan H: Microarray profile of circular RNAs identifies hsa_circ_0014130 as a new circular RNA biomarker in non-small cell lung cancer. Sci Rep 8: 2878, 2018.

7. Li Y, Hu J, Li L, Cai S, Zhang H, Zhu X, Guan G and Dong X: Upregulated circular RNA circ_0016760 indicates unfavorable prognosis in NSCLC and promotes cell progression through miR-1287/GAGE1 axis. Biochem Biophys Res Commun 503: 2089-2094, 2018

8. Chang Y, Yan W, Sun C, Liu Q, Wang J and Wang M: miR-145-5p inhibits epithelial-mesenchymal transition via the JNK signaling pathway by targeting MAP3K1 in non-small cell lung cancer cells. Oncol Lett 14: 6923-6928, 2017.

9. Zhao T, Qian K and Zhang Y: High expression of FGF5 is an independent prognostic factor for poor overall survival and relapse-free survival in lung adenocarcinoma. J Comput Biol 27: 948-957, 2020.

10. Xiao MS and Wilusz JE: An improved method for circular RNA purification using RNase $\mathrm{R}$ that efficiently removes linear RNAs containing G-quadruplexes or structured 3' ends. Nucleic Acids Res 47: 8755-8769, 2019.

11. Chen Y, Peng C, Chen J, Chen D, Yang B, He B, Hu W, Zhang Y, Liu H, Dai L, et al: WTAP facilitates progression of hepatocellular carcinoma via m6A-HuR-dependent epigenetic silencing of ETS1. Mol Cancer 18: 127, 2019.

12. Livak KJ and Schmittgen TD: Analysis of relative gene expression data using real-time quantitative PCR and the 2(-Delta Delta C(T)) method. Methods 25: 402-408, 2001.

13. Zhang X, Yan Y, Lei X, Li A, Zhang H, Dai Z, Li X, Chen W, Lin W, Chen F, et al: Circular RNA alterations are involved in resistance to avian leukosis virus subgroup-J-induced tumor formation in chickens. Oncotarget 8: 34961-34970, 2017.

14. Shi P, Sun J, He B, Song H, Li Z, Kong W, Wang J, Wang J and Xue H: Profiles of differentially expressed circRNAs in esophageal and breast cancer. Cancer Manag Res 10: 2207-2221, 2018.

15. Wang Y, Li Y,He H and Wang F: Circular RNA circ-PRMT5 facilitates non-small cell lung cancer proliferation through upregulating EZH2 via sponging miR-377/382/498. Gene 720: 144099, 2019.

16. Zhang H, Wang X, Hu B, Zhang F, Wei H and Li L: Circular RNA ZFR accelerates non-small cell lung cancer progression by acting as a miR-101-3p sponge to enhance CUL4B expression. Artif Cells Nanomed Biotechnol 47: 3410-3416, 2019.

17. Chen T, Yang Z, Liu C, Wang L, Yang J, Chen L and Li W: Circ 0078767 suppresses non-small-cell lung cancer by protecting RASSF1A expression via sponging miR-330-3p. Cell Prolif 52: e12548, 2019. 
18. Gan TQ, Xie ZC, Tang RX, Zhang TT, Li DY, Li ZY and Chen G: Clinical value of miR-145-5p in NSCLC and potential molecular mechanism exploration: A retrospective study based on GEO, qRT-PCR, and TCGA data. Tumour Biol 39: 1010428317691683, 2017.

19. Lu Q, Shan S, Li Y, Zhu D, Jin W and Ren T: Long noncoding RNA SNHG1 promotes non-small cell lung cancer progression by up-regulating MTDH via sponging miR-145-5p. FASEB J 32: 3957-3967, 2018.

20. Jin M, Ren J, Luo M, You Z, Fang Y, Han Y, Li G and Liu H: Long non-coding RNA JPX correlates with poor prognosis and tumor progression in non-small-cell lung cancer by interacting with miR-145-5p and CCND2. Carcinogenesis 41: 634-645, 2020.

21. Wei CM, Zhao XF, Qiu HB, Ming Z, Liu K and Yan J: The long non-coding RNA PVT1/miR-145-5p/ITGB8 axis regulates cell proliferation, apoptosis, migration and invasion in non-small cell lung cancer cells. Neoplasma 67: 802-812, 2020.
22. Zhou Y, Yu Q, Chu Y, Zhu X, Deng J, Liu Q and Wang Q: Downregulation of fibroblast growth factor 5 inhibits cell growth and invasion of human nonsmall-cell lung cancer cells. J Cell Biochem: Dec 5, 2018 (Epub ahead of print).doi: https://doi. org/10.1002/jcb.28107.

23. Han D, Wang M, Yu Z, Yin L, Liu C, Wang J, Liu Y, Jiang S, Ren $\mathrm{Z}$ and Yin J: FGF5 promotes osteosarcoma cells proliferation via activating MAPK signaling pathway. Cancer Manag Res 11: 6457-6466, 2019.

24. Ghassemi S, Vejdovszky K, Sahin E, Ratzinger L, Schelch K, Mohr T, Peter-Vörösmarty B, Brankovic J, Lackner A, Leopoldi A, et al: FGF5 is expressed in melanoma and enhances malignancy in vitro and in vivo. Oncotarget 8: 87750-87762, 2017

25. Huang Y, Wang $\mathrm{H}$ and Yang Y: Expression of fibroblast growth factor 5 (FGF5) and its influence on survival of breast cancer patients. Med Sci Monit 24: 3524-3530, 2018.

(i) (9) This work is licensed under a Creative Commons Attribution-NonCommercial-NoDerivatives 4.0 International (CC BY-NC-ND 4.0) License. 